\title{
Aqueye optical observations of the Crab Nebula pulsar
}

\author{
C. Germanà ${ }^{1,2}$, L. Zampieri ${ }^{2}$, C. Barbieri ${ }^{3}$, G. Naletto ${ }^{4,5}$, A. Čadež ${ }^{6}$, M. Calvani ${ }^{2}$, M. Barbieri ${ }^{3}$, I. Capraro ${ }^{3,4}$, \\ A. Di Paola ${ }^{7}$, C. Facchinetti ${ }^{3}$, T. Occhipinti ${ }^{3,4}$, A. Possenti ${ }^{8}$, D. Ponikvar ${ }^{6}$, E. Verroi ${ }^{3,4}$, and P. Zoccarato ${ }^{9}$ \\ 1 Departamento de Física, Universidade Federal de Santa Catarina, 88040-900 Florianòpolis, Brazil \\ 2 INAF - Astronomical Observatory of Padova, 35122 Padova, Italy \\ e-mail: luca.zampieri@oapd.inaf.it \\ 3 Department of Physics and Astronomy, University of Padova, 35122 Padova, Italy \\ 4 Department of Information Engineering, University of Padova, 35122 Padova, Italy \\ 5 CNR - IFN UOS Padova LUXOR, 35122 Padova, Italy \\ ${ }^{6}$ Faculty of Mathematics and Physics, University of Ljubljana, 1000 Ljubljana, Slovenia \\ 7 INAF - Astronomical Observatory of Rome, via Frascati 33, 00040 Monteporzio, Rome, Italy \\ 8 INAF - Astronomical Observatory of Cagliari, Str. 54, Loc. Poggio dei Pini, 09012 Capoterra, Sardinia, Italy \\ 9 Curtin University, Department of Spatial Sciences, GNSS Research Centre, GPO Box U1987, Perth, WA 6845, Australia
}

Received 28 December 2011 / Accepted 2 October 2012

\section{ABSTRACT}

\begin{abstract}
Context. We observed the Crab pulsar in October 2008 at the Copernico Telescope in Asiago - Cima Ekar with the optical photon counter Aqueye (the Asiago Quantum Eye), which has the best temporal resolution and accuracy ever achieved in the optical domain (hundreds of picoseconds).

Aims. Our goal was to perform a detailed analysis of the optical period and phase drift of the main peak of the Crab pulsar and compare it with the Jodrell Bank ephemerides.

Methods. We determined the position of the main peak using the steepest zero of the cross-correlation function between the pulsar signal and an accurate optical template.

Results. The pulsar rotational period and period derivative have been measured with great accuracy using observations covering only a two day time interval. The error on the period is $1.7 \mathrm{ps}$, limited only by the statistical uncertainty. Both the rotational frequency and its first derivative agree with those from the Jodrell Bank radio ephemerides archive. We also found evidence that the optical peak precedes the radio peak by $\sim 230 \mu$ s. The distribution of phase residuals of the whole dataset is slightly more scattered than that of a synthetic signal generated as a sequence of pulses distributed in time with the probability proportional to the pulse shape.

Conclusions. The counting statistics and quality of the data allowed us to determine the pulsar period and period derivative with great accuracy in two days only. The time of arrival of the optical peak of the Crab pulsar precedes the radio peak in agreement with what was recently reported in the literature. The distribution of the phase residuals can be approximated with a Gaussian and is consistent with being completely caused by photon noise (for the best data sets).
\end{abstract}

Key words. pulsars: individual: Crab pulsar - techniques: photometric

\section{Introduction}

Since its discovery (Staelin \& Reifenstein 1968; Comella et al. 1969), the pulsar in the Crab nebula has been one of the most often targeted objects in the sky at all wavelengths, from radio to very high energy rays, serving as a test bed for pulsar theories as well as for studying astrophysical non-thermal processes. Optical pulsations were discovered more than 40 years ago (Cocke et al. 1969; Lynds et al. 1969) and the Crab pulsar was indeed the first celestial object to be detected as a pulsating source in the optical band.

The optical light curve of the Crab pulsar has been monitored through the years using a variety of telescopes and instruments (e.g. Słowikowska et al. 2009). The pulse shape is characterized by a double-peak profile, separated in phase by $\sim 140^{\circ}$. The shape is similar throughout the entire electromagnetic spectrum, although the morphological details differ substantially from radio to gamma-rays. Wavelength-dependent changes in the pulsar properties have been reported also by Percival et al. (1993) (peak width and separation larger in the $V$ band than in the UV) and by Fordham et al. (2002). The pulse shape is very stable (e.g. Zampieri et al. 2011) despite the secular decrease in luminosity
(Nasuti et al. 1996) and the presence of glitches and timing noise. Occasionally, weak variations in the shape of the pulse have been observed (Karpov et al. 2007).

Several questions concerning the pulsar engine and the actual geometry of the emission regions are still debated, ranging from the nature and location of the acceleration mechanism to wavelength-dependent variations of the pulse profile, to properties of the giant radio pulses (GRPs). In particular, the study of GRPs is currently a very active field, with deep theoretical implications. So far, GRPs have been observed in a handful of pulsars. However, an analogous optical phenomenon has been observed only at the Crab. The GRPs seem to show a weak correlation with optical pulses (Kuiper et al. 2003; Rots et al. 2004), which are on average $3 \%$ brighter when coincident with GRPs (Shearer et al. 2003). Recent coeval timing at optical and radio wavelengths by Oosterbroek et al. (2008) found a $255 \pm 21 \mu \mathrm{s}$ delay of the radio with respect to optical pulse.

In the past few years we started a monitoring program of the Crab pulsar in the optical band aimed at studying the longterm stability and sub- $\mu$ s structure of its pulse shape, and at performing accurate optical timing of the main peak. By comparing the behavior in other wavebands, especially radio, we aim to 
Table 1. Log of October 2008 Crab pulsar observations performed with Aqueye mounted at the $182 \mathrm{~cm}$ Copernico telescope in Asiago.

\begin{tabular}{llc}
\hline \hline & $\begin{array}{c}\text { Starting time } \\
(\text { UTC) }\end{array}$ & $\begin{array}{c}\text { Duration } \\
(\mathrm{s})\end{array}$ \\
\hline 1 & October 10, 23:45:14 & 898 \\
2 & October 11, 00:05:07 & 1197 \\
3 & October 11, 01:00:22 & 1797 \\
4 & October 11, 01:45:44 & 1797 \\
5 & October 11, 02:23:07 & 1631 \\
6 & October 11, 03:23:46 & 1197 \\
7 & October 11, 23:08:03 & 292 \\
8 & October 11, 23:25:09 & 3597 \\
9 & October 12, 00:54:31 & 1794 \\
10 & October 12, 23:03:59 & 292 \\
11 & October 12, 23:13:57 & 3998 \\
12 & October 13, 00:57:07 & 7194 \\
\hline
\end{tabular}

Notes. The start time of the observations is the GPS integer second, accurate to $\pm 30 \mathrm{~ns}$.

improve the understanding of the geometry of the acceleration site. Observations are performed by means of a very fast single photon-counter instrument, Aqueye, mounted at the $182 \mathrm{~cm}$ Copernico Telescope in Asiago (Barbieri et al. 2009; Zampieri et al. 2011). The design of Aqueye follows that of QuantEYE (the Quantum EYE; Dravins et al. 2005; Barbieri et al. 2009), an instrument specifically tailored for studying rapid optical variability of astrophysical sources with the ESO E-ELT. A second version of the instrument, named Iqueye, has been installed and successfully used at the ESO NTT telescope (Naletto et al. 2009, 2010).

In a preliminary investigation (Zampieri et al. 2011) we concentrated on the pulse-shape stability of the Crab pulsar and found that it is stable at the level of $\sim 1 \%$ on a timescale of 14 years. This result reinforces evidence for decadal stability of the inclination angle between the spin and magnetic axis, and of the thickness of the emission region. Here we present a follow-up investigation reporting accurate phase analysis of optical timing of the main peak and comparing it with radio ephemerides of the Jodrell Bank (JB) radio Observatory (Lyne et al. 1993).

The plan of the paper is as follows. In Sect. 2 we list observations of the Crab pulsar performed with the Aqueye instrument and discuss the barycenterization procedure. In Sect. 3 we illustrate the implementation of the phase analysis. In Sect. 4 our results are presented and compared with radio ephemerides. Section 5 summarizes the conclusions.

\section{Observations}

The Crab pulsar was observed with Aqueye mounted at the $182 \mathrm{~cm}$ Copernico Telescope in Asiago. The observations were performed in 2008 and lasted for three nights, starting from October 10. The sky was clear and seeing conditions fair (1.5 arcsec average). For a timing log of observations, see Table 1. During each observing run we recorded the arrival time of $\sim 0.15-3.6 \times 10^{7}$ photons, time-tagged with a relative time accuracy of $\sim 100$ picoseconds and an absolute precision (referred to UTC) better than 500 ps (for details about the timing accuracy of the acquisition system see Naletto et al. 2009). To our knowledge, this is the most accurate measurement of photon
Table 2. Geocentric coordinates of the $182 \mathrm{~cm}$ Copernico telescope in Asiago.

\begin{tabular}{lcc}
\hline \hline$x$ & $y$ & $z$ \\
$(\mathrm{~m})$ & $(\mathrm{m})$ & $(\mathrm{m})$ \\
\hline 4360966.0 & 892728.1 & 4554543.1 \\
\hline
\end{tabular}

Notes. The $3 \sigma$ uncertainty is $0.3 \mathrm{~m}$.

arrival times from the Crab pulsar ever obtained in the optical band.

The time-tag of each detected photon in the unbinned time series was reduced to the solar system barycentric time using the software Tempo $2^{1}$ (Hobbs et al. 2006; Edwards et al. 2006). The adopted position of the Crab pulsar is that reported in the Jodrell Bank monthly ephemerides (RA 05 $34^{\mathrm{m}} 31.97232^{\mathrm{s}}$, Dec $+22^{\circ} 00^{\prime} 52.0690^{\prime \prime}$ [J2000]), with no correction for proper motion. To perform this conversion the software also needs accurate values of the observatory geocentric coordinates. They were obtained with a GPS receiver, which was connected to an antenna with a length-compensated cable and situated at the dome of the telescope. At least six GPS satellites were used in the positional data acquisition, which typically lasted three hours and was repeated for several days. Finally the position of the antenna was referred to the intersection of the telescope hour angle and declination axes by laser-assisted metrology. We estimate the positional error to be $\sim 30 \mathrm{~cm}$, amply sufficient for the purpose of this paper.

To compare our ephemerides to those reported in the JB Observatory radio archive, we baricentered the time-tags also in the Tempo1 emulation mode. In doing so, we found an error in the actual value of the Roemer delay, caused by some inconsinstency in the Earth configuration files related to the calculation of the polar motion of the Earth ${ }^{2}$. This problem was solved by using updated Earth configuration files loaded from the Tempo2 SCM repository ${ }^{3}$.

\section{Phase analysis}

We analyzed the phase evolution of the main peak of the Crab pulsar using the barycentered event list. A reference period $P_{\text {init }}$ was assumed and the light curve was divided into $n$ seconds long segments. Each segment was then folded over $P_{\text {init }}$ and was binned at $\sim 1 / 300$ in phase. The phase of the main peak was determined by cross-correlating the pulse shape with a template, as summarized in the Appendix. Our method is conceptually similar to the one adopted by Oosterbroek et al. (2008) and is more accurate than previous approaches that were based on fitting the main peak with a simple analytic function like a Lorentzian, Gaussian, or a parabola (see e.g. Oosterbroek et al. 2006), given its asymmetric shape.

Following the standard pulsar spin down model, we describe the phase drift of the main peak with respect to uniform rotation using a third-order polynomial, i.e.,

$\Delta \phi(t)=\phi(t)-\phi^{\prime}(t)=\phi_{0}+\left(v-v_{\text {init }}\right)\left(t-t_{0}\right)+\frac{1}{2} \dot{v}\left(t-t_{0}\right)^{2}+\frac{1}{6} \ddot{v}\left(t-t_{0}\right)^{3}$,

1 http://www.atnf.csiro.au/research/pulsar/ppta/tempo2 2 The Roemer delay computed in Tempo 2 showed an anomalous oscillation at around the time of our observations, reaching a maximum value of $30 \mu$ s (instead of $\lesssim 35 \mathrm{~ns}$; see e.g. Hobbs et al. 2006). This caused a drift of the phase of the main peak of $\sim 1 \mathrm{~ms}$ per day and a lengthening of the rotational period of $\sim 0.4 \mathrm{~ns}$.

3 http://tempo2.cvs. sourceforge.net/viewvc/tempo2/ tempo2/T2runtime/ 
where $t_{0}$ is a reference time, $\phi_{0}=\phi\left(t_{0}\right)$ is the phase of the main peak at $t_{0}, \phi^{\prime}(t)=\left(t-t_{0}\right) / P_{\text {init }}$ is the phase for constant rotation at frequency $v_{\text {init }}=1 / P_{\text {init }}$, and $v, \dot{v}, \ddot{v}$ are the actual rotational frequency and its first and second derivatives, respectively. $P_{\text {init }}$ is chosen in such a way that $\Delta \phi$ varies slowly during the period of observation. For a two-days baseline, the linear and quadratic terms, i.e., the first and second derivatives of the phase, are sufficient to describe the drift, and we can safely neglect the cubic term in Eq. (1). Then, the expression for $\Delta \phi(t)$ (Eq. (1)) becomes of the form

$\psi(t)=\phi_{0}+a\left(t-t_{0}\right)+b\left(t-t_{0}\right)^{2}$,

where $\phi_{0}, a$ (in units of $\mathrm{s}^{-1}$ ) and $b$ (in units of $\mathrm{s}^{-2}$ ) are determined by fitting $\psi(t)$ to the observed phase drift. After determining $\psi(t)$ from the fit, the phase of the main peak is given by

$\phi(t)=\phi^{\prime}(t)+\Delta \phi(t)=v_{\text {init }}\left(t-t_{0}\right)+\psi(t)$.

\subsection{The radio phase from the Jodrell Bank ephemerides archive}

We compared the phase of the Crab pulsar measured by Aqueye with that reported in the radio archive at the JB Observatory ${ }^{4}$ (Lyne et al. 1993). The phases of the JB ephemerides are those of the main peak at infinite frequency at the barycenter of the solar system. The observed barycentric radio phase $\phi_{r}(t)$ is obtained using the values of $v_{r}, \dot{v}_{r}$ and $\ddot{v}_{r}$ nearest to our observing epochs and reported in the archive ${ }^{5}$. The radio phase drift is given by an expression similar to Eq. (1):

$$
\begin{aligned}
\Delta \phi_{r}=\phi_{r}(t)-\phi^{\prime}(t)=\phi_{r, 0}+\left(v_{r}-v_{\text {init }}\right)\left(t-t_{0}\right) & +\frac{1}{2} \dot{v}_{r}\left(t-t_{0}\right)^{2} \\
& +\frac{1}{6} \ddot{v}_{r}\left(t-t_{0}\right)^{3} .
\end{aligned}
$$

The optical phase agrees with that of the radio if the phase drifts inferred from Eqs. (1) and (4) agree. The radio phase $\phi_{r, 0}$ at epoch $t_{0}$ is calculated by means of a quadratic extrapolation starting from the closest value in time reported in the JB radio ephemerides (Oct. 15, 2008) and agrees, within the errors, with the value calculated with a Fortran code available at the JB radio ephemerides website. No known glitch (down to the intensity to which radio monitoring is sensitive to) affected the Crab in the interval of time between the determination of the $\mathrm{Crab}$ radio parameters and the optical observations.

\section{Results}

In Fig. 1 we show the light curve of the Crab pulsar folded over the average spin period for one of the Aqueye observations (see also Zampieri et al. 2011). The light curve includes the contribution of both the pulsar and the nebular background entering the Aqueye pinhole entrance aperture. For the whole observation the counting statistics is large and hence the bin time is shorter than that adopted for the phase analysis, whose typical integration time is a few seconds (see below). The average count rate of the Crab pulsar (all channels) measured by Aqueye is $\sim 5500$ counts $\mathrm{s}^{-1}$. The count rate of the background, estimated from the off-pulse region of the folded light curve (see Fig. 1), is $\sim 4500$ counts $\mathrm{s}^{-1}$, which implies a total number of net source photons of $\sim 2.6 \times 10^{6}$, time-tagged to better than 500 ps with respect to UTC. As we already point out in Zampieri et al. (2011),

\footnotetext{
4 http://www.jb.man.ac.uk/ pulsar/crab.html

5 http://www.jb.man.ac.uk/ pulsar/crab/all.gro
}

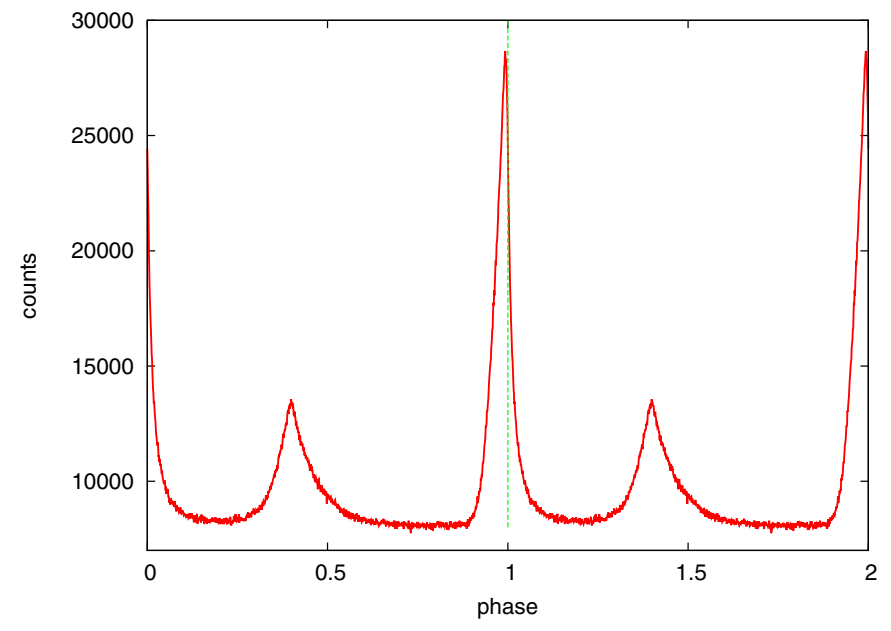

Fig. 1. Folded light curve of the Crab pulsar as a function of phase for Aqueye observation 4 in Table 1 . The folding period and the bin time are $0.0336216417 \mathrm{~s}$ and $33.6 \mu \mathrm{s}$, respectively. The typical double peak profile of the pulse is recognizable. For clarity two rotations of the neutron star are shown. Phase zero/one corresponds to the position of the main peak in the radio band and is marked with a vertical dashed line.

the pulse shape agrees well with the 33 -year-old pulse profile obtained by Groth (1975), as well as with the more recent one by Karpov et al. (2007).

\subsection{Optical phase drift}

In the following we focus on the detailed analysis of the optical phase drift of the main peak of the Crab pulsar and compare it with the behavior observed in the radio using the JB ephemerides. Figure 2 shows the best-fitting parabola to the phase drift of the Crab pulsar measured by Aqueye. The phase of the main peak is calculated using two-second-long intervals. The typical $1-\sigma$ uncertainty on the position of the peak is $\sim 30 \mu \mathrm{s}$. The best fit gives a reduced $\chi^{2} \sim 1.06$. The reference epoch $t_{0}$ is MJD $=54749.0$, while the reference rotational period $P_{\text {init }}=1 / v_{\text {init }}$ used to fold the light curve is $P_{\text {init }}=0.0336216386529 \mathrm{~s}$. The best-fitting parabola of the optical phase drift $\psi(t)$ (Eq. (2)) is

$$
\begin{aligned}
\psi(t)= & (1.021431 \pm 0.000081) \\
& -\left[(3.21329 \pm 0.00011) \times 10^{-5} \mathrm{~s}^{-1}\right]\left(t-t_{0}\right) \\
& +\left[(1.859380 \pm 0.000029) \times 10^{-10} \mathrm{~s}^{-2}\right]\left(t-t_{0}\right)^{2}
\end{aligned}
$$

The uncertainties are the $1-\sigma$ errors for one interesting parameter. Figure 2 also shows the phase residuals remaining after subtracting Eq. (5) from the measured phase drift. The distribution shows a spread of $\lesssim 100 \mu$ s $(\sim 0.003$ cycles $)$ and is rather symmetric around zero (see Fig. 4), testifying that all observations are phase-connected.

The rotational frequecy is $v=\mathrm{d} \phi(t) / \mathrm{d} t$. Thus, inserting Eq. (5) into Eq. (3) and taking the derivative with respect to $t$, we obtain an expression for the rotational frequency $v$ and period $P=1 / v$ at a given (barycentered time) $t$. Table 3 lists the rotational periods of the Crab pulsar measured with Aqueye and those from the JB ephemerides at three reference dates (barycentered MJD = 54750, 54751, 54 752). The differences between optical and radio ranges from $\sim 2$ to $4 \mathrm{ps}$. The statistical error on the optical rotational periods is $1.7 \mathrm{ps}$ ( $1 \sigma$ error). The quoted error from the JB radio archive is $\sim 0.1 \mathrm{ps}$. Thus, the rotational 

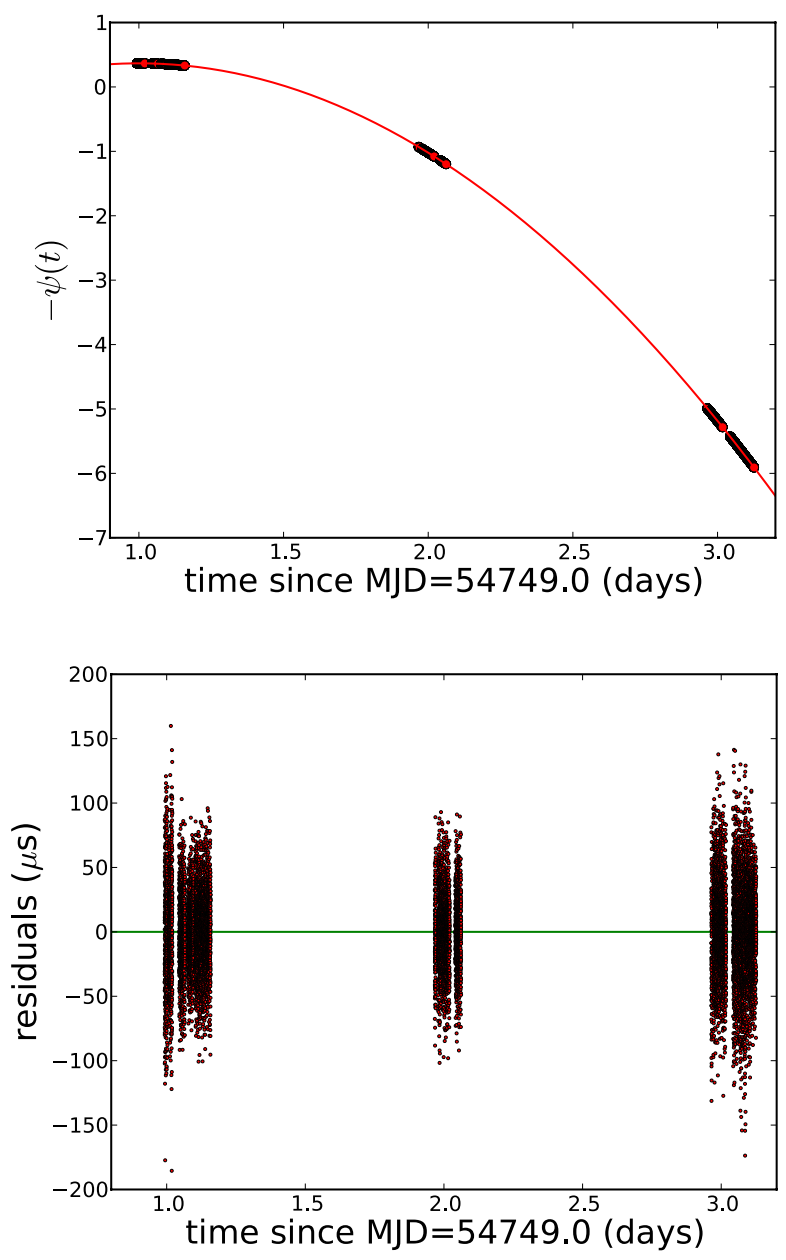

Fig. 2. Top panel: phase drift of the main peak of the Crab pulsar (changed sign) measured during the observing run in Asiago in October 2008. The (red) curve is the best-fitting parabola (Eq. (2)). The reference epoch $t_{0}$ is MJD $=54749$.0, while the reference rotational period is $P_{\text {init }}=0.0336216386529$ s. Bottom panel: phase residuals (in $\mu \mathrm{s}$ ) after subtracting the best-fitting parabola from the phase-drift.

periods calculated by Aqueye agree within the statistical error with those derived from radio measurements. The measurements of the first derivative of the rotational frequency $\dot{v}$ also agree within the errors: $\dot{v}_{\text {Aqueye }}=3.71876 \times 10^{-10} \pm 6 \times 10^{-15} \mathrm{~s}^{-2}$ and $\dot{v}_{\mathrm{JB}}=3.718655 \times 10^{-10} \pm 2 \times 10^{-16} \mathrm{~s}^{-2}$.

We emphasize that to compare our data with those of the JB radio ephemerides, we barycentered the time tags of the optical photons in Tempo 2 using the Tempo/Tempo 1 emulation mode. This is because, for historical reasons, the $\mathrm{JB}$ radio ephemerides are calculated using Tempo, which is the older version of the software used for barycentering. The systems of time adopted in the two packages are different. Tempo 2 uses the barycentric coordinate time (TCB), while Tempo uses the barycentric dynamical time (TDB) ${ }^{6}$. In Table 4 we report rotational periods after barycentering with

\footnotetext{
6 TCB is a coordinate time that refers to the barycenter of the solar system, synchronized with the proper time of a distant observer comoving with it. The system adopted in Tempo1 is the barycentric dynamical time (TDB), effectively measured in units that differ subtly from the conventional SI second (Hobbs et al. 2006). It is as if the time dilation effects were not correctly accounted for using TDB units, so that, for example, rotational periods in the TDB system are systematically shorter than the TCB ones.
}

Table 3. Rotational periods of the Crab pulsar measured by Aqueye in 2008 compared to those reported in the Jodrell Bank radio ephemerides.

\begin{tabular}{lcc}
\hline \hline MJD $^{a}$ & $\begin{array}{c}P(\text { Aqueye })^{b} \\
(\mathrm{~s})\end{array}$ & $\begin{array}{c}P(\mathrm{JB}) \\
(\mathrm{s})\end{array}$ \\
\hline 54750.0 & 0.033621638649 & 0.033621638653 \\
54751.0 & 0.033621674970 & 0.033621674973 \\
54752.0 & 0.033621711290 & 0.033621711292 \\
\hline
\end{tabular}

Notes. The time tags were barycentered in Tempol emulation mode. (a) MJD at the solar system barycenter (Tempo1 mode). ${ }^{(b)} \sigma_{\mathrm{P}}=1.7 \mathrm{ps}$ (68\% statistical error).

Table 4. Rotational periods of the Crab pulsar measured by Aqueye in October 2008.

\begin{tabular}{cc}
\hline \hline MJD $^{a}$ & $\begin{array}{c}P(\text { Aqueye })^{b} \\
(\mathrm{~s})\end{array}$ \\
\hline 54750.0 & 0.033621639166 \\
54751.0 & 0.033621675484 \\
54752.0 & 0.033621711803 \\
\hline
\end{tabular}

Notes. The time tags were barycentered in Tempo 2 (TCB units). ${ }^{(a)} \mathrm{MJD}$ at the solar system barycenter (Tempo2 mode). ${ }^{(b)} \sigma_{\mathrm{P}}=1.7 \mathrm{ps}$ (68\% statistical error).

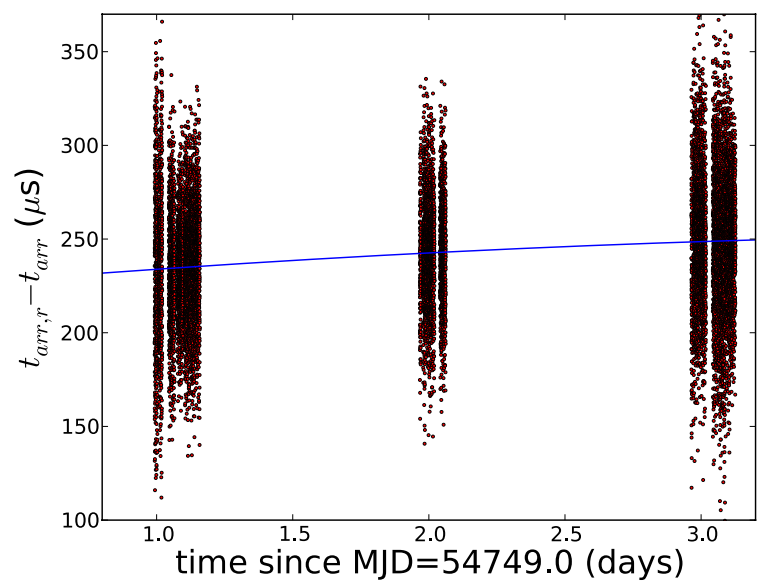

Fig. 3. Difference between the optical and radio time of arrival of the main peak of the Crab pulsar. The optical peak precedes the radio peak by $\sim 230 \mu \mathrm{s}$ (at MJD $=54750$, epoch of the first observation). The (blue) line is the radio-optical drift, which is consistent with zero within the errors (see text).

Tempo2 in TCB units. Although there are other differences between the Tempo/Tempo1 mode setup used for calculating the JB ephemerides of the Crab and the full Tempo 2 mode (as for example the adopted solar system ephemerides), the main difference between rotational periods reported in Tables 3 and 4 is due to the use of TCB units (SI units) instead of TDB. The rotational periods of the $\mathrm{Crab}$ are $\sim 0.5 \mathrm{~ns}$ longer than those measured using Tempo1 in TDB units. If we take the ratio of the periods in Table 4 (TCB units) to those in the second column of Table 3 (TDB units) we find that the ratio of the two time units is $K \sim 1+1.53 \times 10^{-8} \pm 1.3 \times 10^{-10}$, consistent with the value reported by Irwin \& Fukushima (1999) and Hobbs et al. (2006). The constant $K$ sums up a contribution from the linear term of the Einstein delay, $L_{C}$, and another term from the gravitational plus spin potential of the Earth, $L_{\mathrm{G}}$. Thanks to its timing capability and performances, Aqueye shows the occurrence of the corrections $L_{\mathrm{C}}$ and $L_{\mathrm{G}}$ to the pulsar spin period in only two days of data taking on the Crab pulsar. 


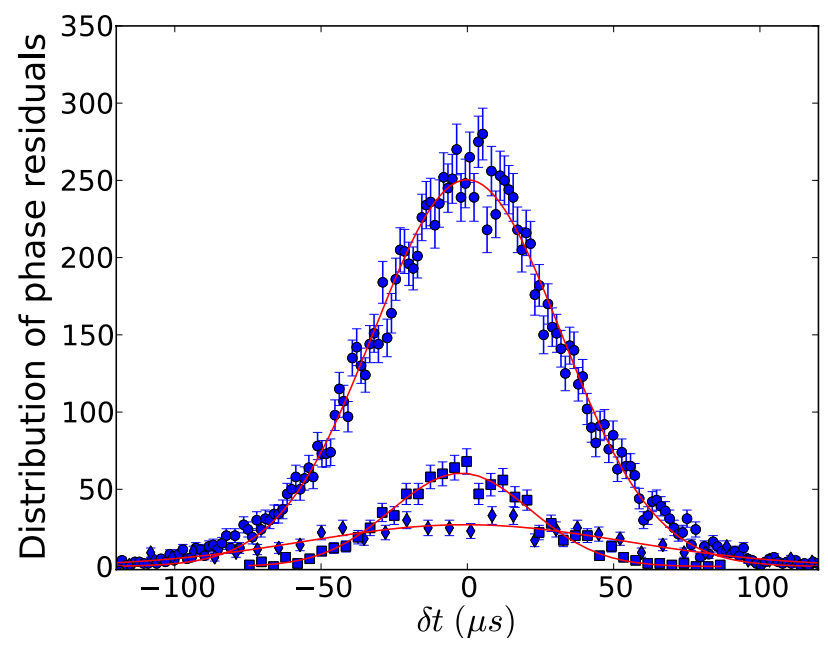

Fig. 4. Residual distribution of the optical phase of the main pulse around the best fit shown in Fig. 2. Each point represents the number of phase residuals $N_{i}$ in the $i$ th bin. The error bar on each bin is $\sqrt{N_{i}}$. The three datasets refer to all observations (circles), to observation 4 (squares) and observation 1 (diamonds). The bin widths are $1.5 \mu \mathrm{s}$, 4.1 $\mu \mathrm{s}$, and $7.3 \mu \mathrm{s}$. The (red) solid lines superimposed to each dataset represent the best-fitting Gaussian with $\sigma \sim 32 \mu \mathrm{s}$ (circles), $\sigma \sim 24 \mu \mathrm{s}$ (squares) and $\sigma \sim 54 \mu$ s (diamonds).

\subsection{Radio-optical delay}

The time of arrival at the detector of the first pulse of the light curve after a certain epoch $t$ is $t_{\text {arr }}=\phi P_{\text {init }}$, where $\phi$ is the phase defined in Sect. 3. Figure 3 shows the difference between the optical time of arrival of the main peak of the Crab pulsar and the radio peak determined from the JB radio ephemerides. The dispersion measure at around the epoch of the Aqueye observations was $56.7842 \mathrm{pc} \mathrm{cm}^{-3}$ (Oct. 15, 2008). We find that the optical peak precedes the radio one. Taking into account the uncertainty on the time of arrival quoted in the JB $\operatorname{archive}^{7}(\sim 60 \mu \mathrm{s})$ and the errors from the fit, the time difference is $\sim 230 \pm 60 \mu \mathrm{s}$ at $\mathrm{MJD}=54750$, with a drift of $\simeq 7 \mu \mathrm{s} /$ day. While within the errors the drift is consistent with zero, the difference in the arrival times is significant. The optical peak preceds the radio peak, in agreement with what was found previously by Sanwal (1999), Oosterbroek et al. (2006), and Oosterbroek et al. (2008), the latter obtained using simultaneous optical and radio observations. Our value of the radio delay is also consistent with the recent measurement performed by our group with Iqueye (178 $\mu$ s; Collins et al. 2012), but it is higher than the one reported in Shearer et al. (2003). The uncertainty on our measurement is dominated by the error on the radio ephmerides, and can be further reduced in future using simultaneous radio-optical observations.

\subsection{Phase noise}

Figure 4 shows the distribution of the phase residuals in Fig. 2. The distribution is fit with a Gaussian and gives a reduced $\chi^{2} \sim 0.9$ for all observations. The Gaussian has $\sigma \sim 32 \mu \mathrm{s}$, consistent with the error bar of each measured phase (see Sect. 3). This indicates that, with the present accuracy, the phase noise of the Crab pulsar observed with Aqueye can be approximated with a Gaussian. However, the distribution of phase residuals

\footnotetext{
7 http://www.jb.man.ac.uk/ pulsar/crab/crab2.txt
}

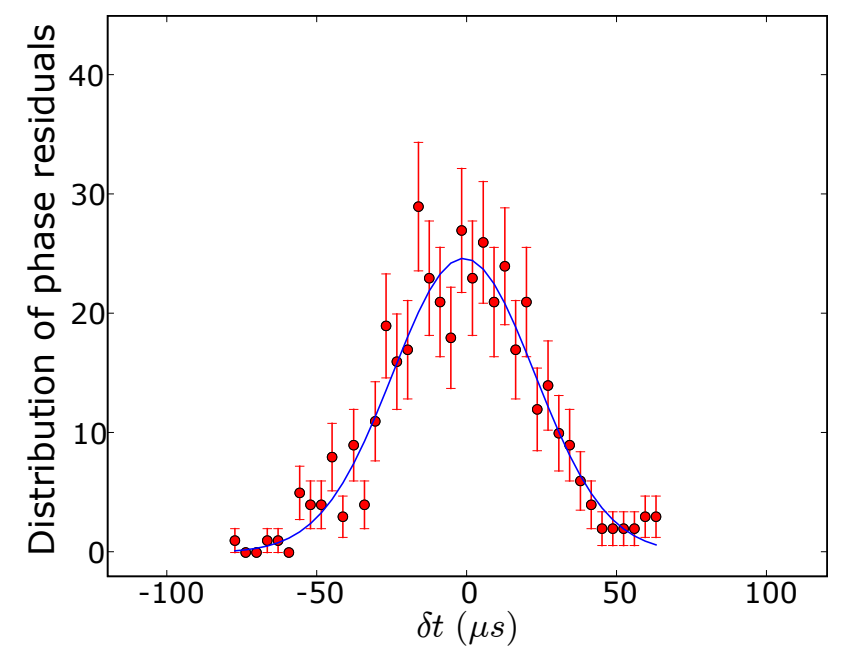

Fig. 5. Same as Fig. 4 for a simulated signal with superimposed random noise (see text for details). The assumed count rate is the average countrate measured by Aqueye. The bin width is $3.6 \mu \mathrm{s}$. The distribution is fit by a Gaussian with $\sigma \sim 24 \mu$ s.

in different observations appears to have different widths. The distributions with the smallest (obs. 4) and largest (obs. 1) widths are also shown in Fig. 4 for comparison. This suggests the existence of additional errors in the data chain of some observations, possibly induced by signal loss (e.g. clouds or telescope tracking errors).

We compared the observed phase residuals with those obtained from a synthetic signal generated as a sequence of pulses distributed in time with the probability proportional to the pulse shape (so that the average count rate and background level are those of the Crab pulsar observed with Aqueye). The signal has superimposed random noise and lasts $\sim 850 \mathrm{~s}$. Figure 5 shows the residuals distribution of the simulated signal. The distribution is clearly Gaussian but with $\sigma \sim 24 \mu \mathrm{s}$. This is smaller than the total phase noise distribution in Fig. 4. However, as mentioned above, the observations have different distributions widths. Some of them are comparable to (obs. 4) or marginally larger (obs. 6 and 7) than that inferred from the simulation, while some others have more outliers. It is thus possible that the outliers are caused by errors in the data chain, induced by the poorer quality of the dataset. For the best dataset (obs. 4; see Fig. 4), the measured width of the phase residual distribution appears to approach the theoretical expectations for phase noise induced by pure photon statistics.

\section{Discussion and conclusions}

We observed the Crab pulsar with the photon counting instrument Aqueye, mounted at the $182 \mathrm{~cm}$ Copernico telescope in Asiago, during the nights of October 10-13, 2008. The counting statistics and quality of the data allowed us to monitor the phase of the main peak of the Crab pulsar over $2 \mathrm{~s}$ long intervals and to determine the pulsar rotational period and period derivative with great accuracy, using observations covering only a two day interval in time. The statistical error on the period inferred from a fit of the pulsar phase drift is on the order of a few picoseconds. The measurements of the period and period derivative agree within the statistical error with those inferred from the JB ephemerides.

We also found that the time of arrival of the optical peak of the Crab pulsar precedes the radio peak (with the latter inferred 
from the JB radio ephemerides), in agreement with previous findings (Sanwal 1999; Shearer et al. 2003). The actual value of the radio delay, $\sim 230 \mu \mathrm{s}$, agrees with the most accurate measurement previously reported in the literature by Oosterbroek et al. (2008). Previous measurements performed using fitting functions of the main peak are less precise. In this case, as pointed by Oosterbroek et al. (2006) and Collins et al. (2012), the phase of the main peak depends on the chosen fitting function, introducing a systematic difference caused by the intrinsic asymmetric shape of the peak. Assuming a Gaussian or Lorentzian fit, different values of the phase of the peak are obtained for different fitting ranges around it. Using a 0.1, 0.05, 0.02 phase interval to the right and to the left of the peak, the difference in position is $247 \mu \mathrm{s}, 230 \mu \mathrm{s}, 134 \mu \mathrm{s}$ for the Gaussian fit and $197 \mu \mathrm{s}$, $197 \mu \mathrm{s}, 114 \mu \mathrm{s}$ for the Lorentzian. Furthermore, it is important to note that the correct estimate of the radio-optical time delay can be obtained only from simultaneous optical and radio observations using similar procedures for the barycenterization and the analysis (e.g. Collins et al. 2012).

As pointed out by Oosterbroek et al. (2006), a time delay of the radio peak of $\sim 230 \mu$ s could have two different interpretations. The emission region of the optical radiation is (a) higher in the magnetosphere $(\sim 70 \mathrm{~km})$ than the radio emission; (b) located at a different angle $\left(\sim 2.5^{0}\right)$ with respect to the radio emission.

We also studied the phase noise distribution of the Crab pulsar observed with Aqueye and found that, with the present accuracy, it can be modeled as a Gaussian. The width of the distribution of the entire dataset is slightly larger than that induced by pure photon random noise from a synthetic signal having the same pulse shape, average count rate, and background level as the Crab pulsar observed with Aqueye. However, residual systematic errors in the data chain may be present in a subset of observations that tend to broaden the distribution. Indeed, the distribution of the best batch of data, i.e. that with the smaller width, is consistent with that induced by photon statistics. While the observed broadening seems to be caused mostly by the deterioration in the quality of some observations, the existence of a smaller source of phase noise, possibly related to the intrinsic pulsar mechanism, cannot be ruled out at present and needs to be carefully investigated with future observations.

Acknowledgements. We thank the referee for his/her constructive criticisms that helped to improve our paper. We also would like to thank Alessandro Patruno (Astronomical Institute, University of Amsterdam) for useful discussions. We acknowledge the use of the Crab pulsar radio ephemerides available at the web site of the Jodrell Bank radio Observatory (http://www . jb.man .ac .uk/ pulsar/crab.html; Lyne et al. 1993). This work has been partly supported by the University of Padova, by the Italian Ministry of University MIUR through the program PRIN 2006 and by the Program of Excellence 2006 Fondazione CARIPARO.

\section{Appendix A: Calculating the phase of the main peak}

We start by folding the light curve over a fixed reference period $P_{\text {init }}$, corresponding to the period of the pulsar some time during the complete observing run.

The phase drift of the main peak $\psi$ (see Sect. 3) is obtained by finding the maximum of the correlation function $K$ between the actual pulsar signal $P(\phi(t))$ and a template $S\left(t / P_{\text {init }}-x\right)$ with a time dependence closely resembling the actual pulsar signal, but with a varying phase $x$. The function $S(x)$ is a periodic

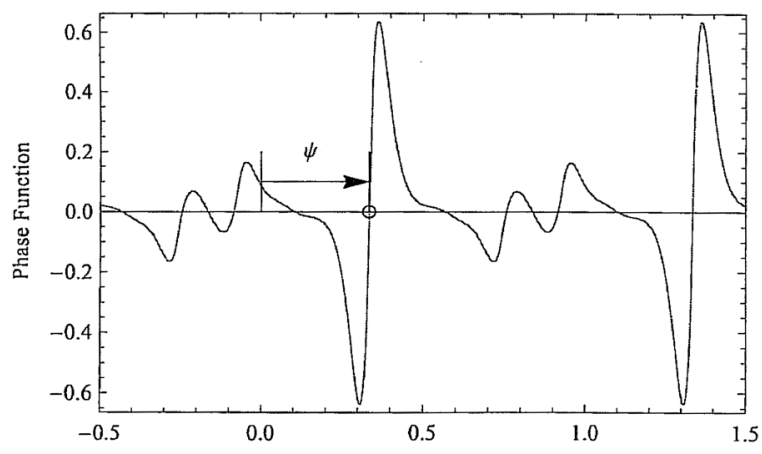

Fig. A.1. Example of the behavior of $K^{\prime}\left(t_{1}, x\right)$.

function with period 1, obtained as a smoothed version of the whole dataset (using 3504 data points in phase):

$K\left(t_{1}, x\right)=\int_{t_{1}}^{t_{1}+\Delta t} P(\phi(t)) S\left(t / P_{\text {init }}-x\right) \mathrm{d} t$,

where $\Delta t$ is the averaging time (usually $2 \mathrm{~s}$ ) and $t_{1}+\Delta t / 2$ the mid point of the averaging interval. After taking the derivative of $K$ with respect to $x$, we obtain

$K^{\prime}\left(t_{1}, x\right)=-\int_{t_{1}}^{t_{1}+\Delta t} P(\phi(t)) S^{\prime}\left(t / P_{\text {init }}-x\right) \mathrm{d} t$.

The value of $\psi$ is obtained taking $K^{\prime}\left(t_{1}, x\right)=0$ and considering only the zero with the steepest crossing (see Fig. A.1).

We select the steepest zero crossing because we are looking for the maximum that corresponds to the sharpest peak of $K$. Let $f(x)$ be a differentiable function with many extrema $x_{1}$, $x_{2}, \ldots$ Then, in the vicinity of each peak it can be expanded as $f(x)=f\left(x_{k}\right)+(1 / 2) f^{\prime \prime}\left(x_{k}\right)\left(x-x_{k}\right)^{2}+\ldots$ The function has maximum at $x_{k}$ if $f^{\prime \prime}\left(x_{k}\right)<0$ and a minimum if $f^{\prime \prime}\left(x_{k}\right)>0 . f^{\prime}(x)$ has a zero at $x_{k}$ and the slope of $f^{\prime}\left(x_{k}\right)$ is $f^{\prime \prime}\left(x_{k}\right)$. The absolute value of $f^{\prime \prime}\left(x_{k}\right)=f\left(x_{k}\right) / w^{2}$, where $w$ is the width of the peak of the function at half maximum. Since it is unlikely that a correlation function would have a small very narrow peak that would make $\left|f^{\prime \prime}\right|$ larger than the main peak, one concludes that the highest peak also has the highest value of $\left|f^{\prime \prime}\right|$ and hence the steepest $\left|f^{\prime}\right|$.

\section{References}

Barbieri, C., Naletto, G., Occhipinti, T., et al. 2009, J. Mod. Opt., 56, 261 Beskin, G., Komarova, V., \& Plokhotnichenko, V. 2000, Nucl. Phys. B Proc. Suppl., 80, C1103

Bolton, J. G., Stanley, G. J., \& Slee, O. B. 1949, Nature, 164, 101

Bowyer, S., Byram, E. T., Chubb, T. A., \& Friedman, H. 1964, Science, 146, 912 Čadež, A., \& Galičič, M. 1996, A\&A, 306, 443

Čadež, A., Galičič, M., \& Calvani, M. 1997, A\&A, 324, 1005

Čadež, A., Vidrih, S., Galičič, M., \& Carramiñana, A. 2001, A\&A, 366, 930 Carramiñana, A., Čadež, A., \& Zwitter, T. 2000, ApJ, 542, 974

Cocke, W. J., Disney, M. J., \& Taylor, D. J. 1969, Nature, 221, 525

Collins, S., Shearer, A., Stappers, B., et al. 2012, IAU Symp., 285, 296

Comella, J. M., Craft, H. D., Lovelace, R. V. E., \& Sutton, J. M. 1969, Nature, 221,453

Dean, A. J., Clark, D. J., Stephen, J. B., et al. 2008, Science, 321, 1183

Dravins, D., Barbieri, C., Fosbury, R. A. E., et al. 2005, Proceedings from meeting Instrumentation for Extremely Large Telescopes, held at Ringberg Castle, July 2005, ed. T. Herbst [arXiv: astro-ph/0511027]

Edwards, R. T., Hobbs, G. B., \& Manchester, R. N. 2006, MNRAS, 372, 1549

Fordham, J. L. A., Vranesevic, N., Carramiñana, A., et al. 2002, ApJ, 581, 485 
C. Germanà et al.: Crab pulsar optical observations with Aqueye

Groth, E. J. 1975, ApJ, 200, 278

Haymes, R. C., Ellis, D. V., Fishman, G. J., Kurfess, J. D., \& Tucker, W. H. 1968, ApJ, 151, L9

Hewish, A., Bell, S. J., Pilkington, J. D. H., Scott, P. F., \& Collins, R. A. 1968, Nature, 217, 709

Hobbs, G. B., Edwards, R. T., \& Manchester, R. N. 2006, MNRAS, 369, 655

Irwin, A. W., \& Fukushima, T. 1999, A\&A, 348, 642

Kanbach, G., Słowikowska, A., Kellner, S., \& Steinle, H. 2005, in Astrophysical

Sources of High Energy Particles and Radiation, eds. T. Bulik, B. Rudak, \&

G. Madejski, AIP Conf. Ser., 801, 306

Karpov, S., Beskin, G., Biryukov, A., et al. 2007, Ap\&SS, 308, 595

Kuiper, L., Hermsen, W., Walter, R., \& Foschini, L. 2003, A\&A, 411, L31

Larsson, S. 1996, A\&AS, 117, 197

Lundqvist, P., Sollerman, J., Gull, T. R., et al. 1999, in BAAS, 31, 903

Lynds, R., Maran, S. P., \& Trumbo, D. E. 1969, ApJ, 155, L121
Lyne, A. G., Pritchard, R. S., \& Graham-Smith, F. 1993, MNRAS, 265, 1003 Minkowski, R. 1942, ApJ, 96, 199

Naletto, G., Barbieri, C., Occhipinti, T., et al. 2009, A\&A, 508, 531

Naletto, G., Barbieri, C., Verroi, E., et al. 2010, Proc. SPIE, 7735

Nasuti, F. P., Mignani, R., Caraveo, P. A., \& Bignami, G. F. 1996, A\&A, 314, 849

Oosterbroek, T., de Bruijne, J. H. J., Martin, D., et al. 2006, A\&A, 456, 283

Oosterbroek, T., Cognard, I., Golden, A., et al. 2008, A\&A, 488, 271

Percival, J. W., Biggs, J. D., Dolan, J. F., et al. 1993, ApJ, 407, 276

Rots, A. H., Jahoda, K., \& Lyne, A. G. 2004, ApJ, 605, L129

Sanwal, D. 1999, Ph.D. Thesis, Univ. Texas at Austin

Shearer, A., Stappers, B., O'Connor, P., et al. 2003, Science, 301, 493

Słowikowska, A., Kanbach, G., Kramer, M., \& Stefanescu, A. 2009, MNRAS 397, 103

Staelin, D. H. \& Reifenstein, III, E. C. 1968, Science, 162, 1481

Zampieri, L., Germaná, C., Barbieri, C., et al. 2011, Adv. Space Res., 47, 365 